\title{
A Comparative Study of Memory Structures for DSM Systems on Wireless Environments"
}

\author{
Hsiao-Hsi Wang, Kuang-Jui Wang, Chien-Lung Chou, \\ Ssu-Hsuan Lu, and Kuan-Ching Li \\ Parallel and Distributed Processing Center \\ Dept. of Computer Science and Information Management \\ Providence University Shalu, Taichung 43301 Taiwan \\ \{hhwang, g9371014, g9371001, g9234024, kuancli\}@pu.edu.tw
}

\begin{abstract}
In recent years, wireless networking has become more popular than ever. Distributed shared memory (DSM) combines computer hardware resources in order to achieve the efficiency and high performance provided by parallel computing. Unfortunately, the major overhead of DSM software is the communication time, especially in wireless network environments. In this paper, we have implemented three memory structures over JIAJIA DSM system on wireless network, and then analyzed their performance. From experimental results, we could find out the relation between communication time and memory layouts. In addition, we have also discovered relationship between characteristics of application programs and memory structures. Experimental results of five well-known benchmark applications show that a suitable memory layout can effectively reduce communication overhead in wireless network. We have analyzed advantages and disadvantages of these memory structures, to improve future designs of wireless DSM systems.
\end{abstract}

\section{Introduction}

Distributed Shared Memory (DSM) software system is an excellent technique and easy alternative for concurrent computing, since computers can exchange data via high speed network to achieve higher performance. In original DSM system architectures, the common shared memory is made by combining all computing nodes' local memories. The home pages are located on all nodes, so nodes often need to access data in remote nodes. This induces remote access latencies, especially when using wireless networking technologies. Due to a number of limitations present in wireless computing environments, such as narrow bandwidth in wireless communication, unstable connectivity and data synchronization in mobile terminals are present.

S. Yokoyama et al. proposed a memory management architecture named Memory Management Architecture for Mobile Computing Environment (MMM) [1]. From initial concepts present in MMM, we have some ideas. We built a wireless DSM system over JIAJIA DSM software [2] and compare three different layouts of home

\footnotetext{
This research is supported by National Science Council (NSC), Taiwan, under grant no. NSC
} 94-2745-E-126-002-URD. 
pages, which are Hybrid Memory Structure (HMS), Centralized Memory Structure (CMS) and Distributed Memory Structure (DMS). We expect to find out main drawbacks when using wireless network and to improve these problems. In addition, we also expect to find out some relation between the characteristics of application programs and memory structures, such as the bandwidth, packet losing rate, and disconnection.

The remaining of this paper is organized as follows. In section 2, we compare the difference among three memory layouts. In section 3, we bring up experimental performance evaluation and finally, some conclusions and future works in section 4 .

\section{Memory Structures}

In this section, we will describe three memory structures and compare differences between them, as show in Figure 1.

(A). Distributed Memory Structure (DMS): In JIAJIA, the pages are initially distributed among all nodes in the original memory layout. If any of nodes occurs page faults and needs to get pages from remote nodes, nodes will communicate with each other. If there are many nodes that need to send data at the same time, it maybe occur competition for network bandwidth or data retransmission. Due to the fact that bandwidth of wireless network is limited, it may not be suitable for wireless DSM system.

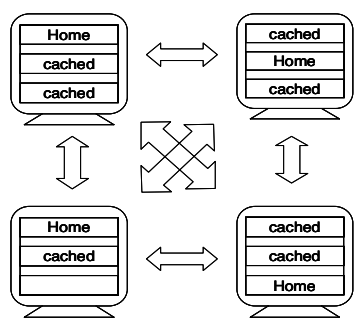

(A)

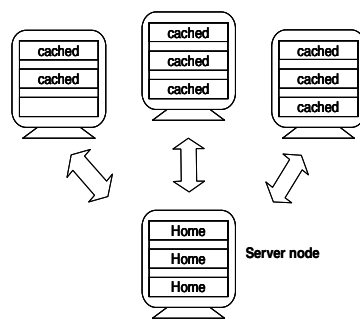

(B)

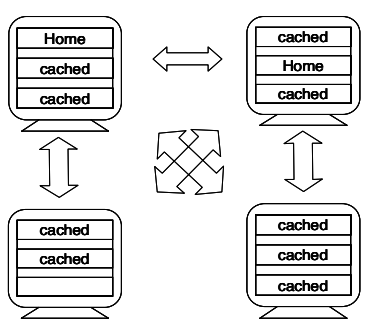

(C)

Fig. 1. (A). Distributed Memory Structure. (B). Centralized Memory Structure. (C). Hybrid Memory Structure.

(B). Centralized Memory Structure (CMS): We changed the original memory layout in JIAJIA software system, according to the design of MMM. If some node needs to access data, it will request to server. The server node maintains the consistency of the common memory areas and provides pages for client. Other nodes only have cache pages. In other words, when the page fault occurs on server node it only takes pages from itself, as also the times of communication will reduce, since the communication is simpler than DMS.

(C). Hybrid Memory Structure (HMS): In Hybrid Memory Structure (HMS), half of nodes have home pages and caches and the other nodes just have caches. In order to 
reduce communication overhead on server node, but still can reduce the times of communication, so we have an idea that located home pages on half of nodes. It can have better load balancing between communication and the percentage of data access hits.

\section{Performance Evaluation}

We have built a wireless DSM system platform for our experiments, and the hardware computing platform we used for our investigation is constructed using 4 PCs, each containing one Intel P4 3GHz CPU, 256 MB DDR memory, ASUS USB wireless network adapter 54MB/s and Fedora Core 3 OS with kernel version 2.6.9. We evaluate the performance of DMS, CMS, and HMS structures running five different parallel applications: IS from NAS [3], LU from SPLASH2 [4], Merge, SOR, and TSP. Table 1 shows the problem size and characteristics of these applications.

Table 1. Characteristics of Benchmark Applications

\begin{tabular}{|c|c|c|c|c|}
\hline Application & Size & Memory (MB) & Barriers & Locks \\
\hline IS & $2^{24}, 2^{10}, 10$ & 0.32 & 32 & 40 \\
\hline LU & $1024 * 1024,32$ & 10.28 & 68 & 0 \\
\hline Merge & $200 * 7500$ & 7.36 & 5 & 0 \\
\hline SOR & $512,256,50$ & 10.24 & 101 & 0 \\
\hline TSP & 19 (cities) & 0.99 & 2 & 687 \\
\hline
\end{tabular}

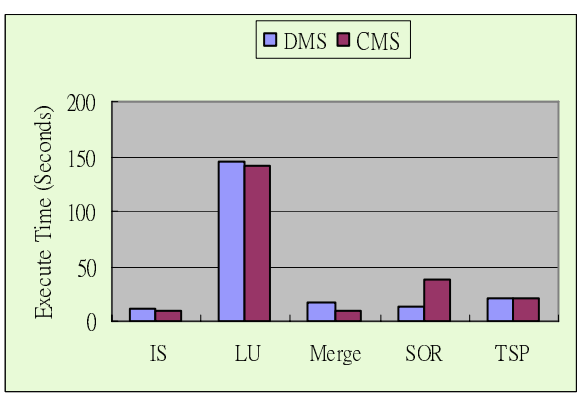

(A)

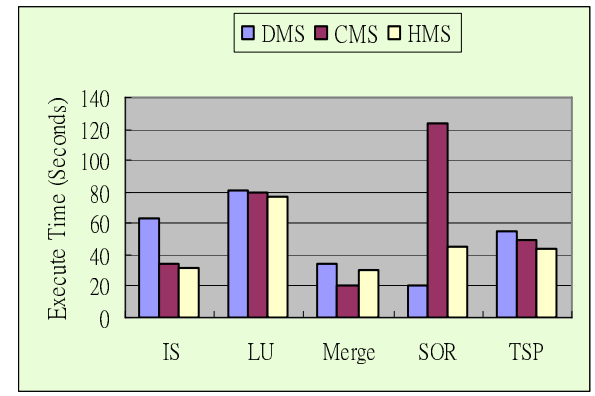

(B)

Fig. 2. Execution Time of Parallel Applications over Wireless DSM Platform. (A). Using 2 Wireless Computing Nodes (B). Using 4 Wireless Computing Nodes.

Figure 2(A) shows the execution time of original JIAJIA and CMS on two wireless nodes. We can see that some applications like IS and Merge on CMS can have better performance than that on DMS. If page faults happen, it will need to transmit the data among every node on DMS, since the bandwidth is limited in the wireless environment. If there are too many nodes transmitting data at the same time, it will cause bandwidth competition, data loss and data retransmission. Therefore, the performance will degrade rapidly, and this phenomenon can be seen obviously under four nodes DSM environment. 
Additionally, we find that the characteristics of the application programs are important factors, such as Merge and SOR. In SOR, the original structure initially distributes red and block arrays across all nodes, but CMS allocates all shared data in one node. CMS opposites to the characteristic of SOR itself and causes the hits rate dropping and the communication time raising a lot. The characteristic of Merge is completely opposite to SOR. The best performance of Merge is in CMS and the worst performance of SOR is also in CMS, as in Figure 2(B).

\section{Conclusions and Future Work}

According to experimental results, we believe that is very sensitive the relation between the home location and performance. It is essential to locate the home pages correctly and the CMS can reduce the cost of communication. Under the wireless network environment, it is not reliable as in wired network, as it has narrow bandwidth. As large amount of data need to be transmitted, packets easy to collide as also to lose in wireless network. If we can improve the problems in this respect, it will improve global DSM system efficiency.

In addition, there exist close relation between the characteristics of applications and the percentage of access hit rates. It may cause performance come down fast if the location of home pages and the access pattern of application program are not suitable. The percentage of hits and increase efficiency can be improved if we can offer a suitable structure for home pages layout.

As future work, we will continue to probe the DSM software system in wireless environment and find out a number of main factors for improvement. We are looking for more effective methods to reduce the amount of message passing, to increase the percentage of hit rates for reducing the burden of the network, and to improve the efficiency of the wireless DSM environment.

\section{References}

[1] S. Yokoyama, T. Mizuno, and T. Watanabe, "A Proposal of a Memory Management Architecture for Mobile Computing Environments," in the Proceedings of Database and Expert Systems Applications, 2000, pp. 28-32.

[2] W. Hu, W. Shi, and Z. Tang, "Reducing System Overheads in Home-Based Software DSMs," in the Proceedings of 13th International and 10th Symposium on Parallel and Distributed Processing, 1999, pp. 167-173.

[3] D. Bailey, J. Barton, T. Lasinski, and H. Simon, "The NAS Parallel Benchmarks," Technical Report 103863, NASA, 1993.

[4] S. Woo, M. Ohara, E. Torrie, J. Singh, and A. Gupta, "The SPLASH-2 Programs: Characterization and Methodological Considerations," in the Proceedings of 22nd Annual International Symposium on Computer Architecture, 1995, pp.24-36. 